\title{
SUPER-REPLICATION IN STOCHASTIC VOLATILITY MODELS UNDER PORTFOLIO CONSTRAINTS
}

\author{
JAKŠA CVITANIĆ, ${ }^{*}$ Columbia University \\ HUYÊN PHAM, ${ }^{* *}$ Université Marne-la-Vallée and CREST \\ NIZAR TOUZI, ${ }^{* * *}$ CEREMADE and CREST
}

\begin{abstract}
We study a financial market with incompleteness arising from two sources: stochastic volatility and portfolio constraints. The latter are given in terms of bounds imposed on the borrowing and short-selling of a 'hedger' in this market, and can be described by a closed convex set $K$. We find explicit characterizations of the minimal price needed to super-replicate European-type contingent claims in this framework. The results depend on whether the volatility is bounded away from zero and/or infinity, and also, on if we have linear dynamics for the stock price process, and whether the volatility process depends on the stock price. We use a previously known representation of the minimal price as a supremum of the prices in the corresponding shadow markets, and we derive a PDE characterization of that representation.
\end{abstract}

Keywords: Stochastic volatility; portfolio constraints; hedging options; viscosity solutions

AMS 1991 Subject Classification: Primary 90A09; 93E20; 60H30

Secondary 60G44; 90A16

\section{Introduction}

We consider a Markovian model of a financial market in which the stock price is a solution of a (possibly nonlinear) stochastic differential equation (SDE), with the volatility coefficient driven by another diffusion process. There are two Brownian motions driving the corresponding SDEs, therefore the market is incomplete. This is a standard way of modeling volatility risk which is not hedgable by investing in the underlying only, and it was used by Hull and White (1987) and Wiggins (1987), amongst others. We are primarily interested in the minimal super-replication cost in this model of a European type option with payoff $g(s)$ namely the cost of the least expensive dominating strategy for the option. Additionally, we also analyse what happens if we impose convex constraints on the portfolio weights of the

Received 8 May 1997; revision received 21 October 1997.

* Postal address: Department of Statistics, Columbia University, 2990 Broadway, New York, NY 10027.

Email address: cj@stat.columbia.edu

** Postal address: Equipe d'Analyse et de Mathématiques Appliquées, Université Marne-la-Vallée, Cité Descartes, 5 Boulevard Descartes, Marne-la-Vallée, Cedex 77454, France.

*** Postal address: CEREMADE, Université Paris Dauphine, Place du Marechal de Lattre de Tassigny, 75775 Paris Cedex 16, France. 
hedging wealth process. The latter problem is solved in Broadie et al. (1998), in the BlackScholes constant volatility framework. They show that the minimal cost for super-replication of $g$ under constraints is given by pricing another option $\hat{g}(s) \geq g(s)$ without the constraints, with $\hat{g}$ appropriately defined, to make the super-replicating portfolio satisfy the constraints. We extend their results to the case of stochastic volatility.

In Section 2 we introduce the model and the constraints. In Section 3 we define the shadow prices corresponding to the incompleteness of the market, and we re-derive a result of Cvitanić and Karatzas (1993), Jouini and Kallal (1995) and El Karoui and Quenez (1995), which gives a lower bound on the minimal super-replication cost as a supremum of Black-Scholes prices over all the associated shadow markets.

In Section 4 we state the main technical result (which is proved in the Appendix), i.e. the cost (price) function is a viscosity supersolution to the Bellman equation associated with the control problem (the use of the viscosity supersolutions approach turns out to be very powerful and elegant in our problem). Next, using those results, in Section 5 we show that when the volatility can reach (in the limit) both zero and infinity, or if it can reach infinity and the payoff $g$ is convex, the minimal super-replication cost (without constraints) is the cost of the minimal buy-and-hold strategy - namely, equal to the concave envelope of $g$. If there are constraints, such that one is allowed to put all the money in the stock, then it is the concave envelope of $\hat{g}$ that is equal to the minimal cost. In Section 6 we consider the case of bounded volatility, and rederive the so-called Barenblatt PDE, suggested as the PDE for obtaining the super-replicating price under stochastic volatility in Avellaneda et al. (1995). Our analysis shows that this gives rise not only to a super-replicating strategy, but also to the least expensive one. Moreover, in the case of linear dynamics, we extend the result to the case with portfolio constraints, simply by substituting $\hat{g}$ instead of $g$ for the terminal condition. Similar results are shown to be valid in the mixed case (unbounded volatility bounded away from zero) in Section 7. Finally, in Section 8 we provide some examples.

Let us also mention that while finishing this paper we learned that, in the case of unbounded volatility and no constraints, Frey and Sin (1999) obtained results similar to ours; however, they use completely different methods and deal with less general payoffs, but more general price processes. Related work also includes El Karoui et al. (1998), and Bergman et al. (1996).

\section{The model}

We consider a financial market which consists of one bank account, with constant price process $B(t)=1$ for all $t \in[0, T]$, and one risky asset with price process evolving according to the following stochastic differential equation:

$$
\begin{aligned}
\frac{\mathrm{d} S(t)}{S(t)} & =\mu(t, S(t), Y(t)) \mathrm{d} t+\sigma_{1}(t, S(t), Y(t)) \mathrm{d} W_{1}(t)+\sigma_{2}(t, S(t), Y(t)) \mathrm{d} W_{2}(t), \\
\mathrm{d} Y(t) & =\eta(t, S(t), Y(t)) \mathrm{d} t+\gamma(t, S(t), Y(t)) \mathrm{d} W_{2}(t) .
\end{aligned}
$$

Here $W=\left(W_{1}, W_{2}\right)$ is a standard Brownian motion in $\mathbb{R}^{2}$ defined on a complete probability space $(\Omega, \mathcal{F}, P)$. We shall denote by $\{\mathcal{F}(t)\}$ the $P$-augmentation of the filtration generated by $W$. The assumption that the interest rate of the bank account is zero could, as usual, be easily dispensed with, by discounting. Throughout this paper, we make the usual standing assumptions on the coefficients of the last SDE in order to ensure the existence of a unique strong solution $\left\{\left(S_{t}, Y_{t}\right), 0 \leq t \leq T\right\}$, given the initial condition, that all coefficients are continuous in $(t, s, y)$ and satisfy for all $t \in[0, T]$ and $(s, y),\left(s^{\prime}, y^{\prime}\right) \in \mathbb{R}_{+}^{*} \times \mathbb{R}$, 


$$
\begin{aligned}
& \sum_{i=1,2}\left(\left|s \sigma_{i}(t, s, y)-s^{\prime} \sigma_{i}\left(t, s^{\prime}, y^{\prime}\right)\right|+\left|\gamma(t, s, y)-\gamma\left(t, s^{\prime}, y^{\prime}\right)\right|\right. \\
& \left.\quad+\left|s \mu(t, s, y)-s^{\prime} \mu\left(t, s^{\prime}, y^{\prime}\right)\right|+\left|\eta(t, s, y)-\eta\left(t, s^{\prime}, y^{\prime}\right)\right|\right) \leq C\left(\left|s-s^{\prime}\right|+\left|y-y^{\prime}\right|\right),
\end{aligned}
$$

for some positive constant $C$. We also assume

$$
\forall(t, s, y) \in[0, T] \times \mathbb{R}_{+}^{*} \times \mathbb{R}, \quad \sigma_{1}(t, s, y)>0 \text { and } \gamma(t, s, y)>0 .
$$

Moreover, for simplicity, we assume that for any $y \in \mathbb{R}$, there is a positive probability that the process $Y$ will reach $y$ before time $T$.

Remark 2.1. Because our goal is mostly to illustrate the extreme behavior of the minimal super-replicating price that can happen in stochastic volatility models, we do not always aim at the most general assumptions possible. We could assume Lipschitz conditions in $\log s$ on the coefficients in (2.1) and (2.2), for example, and the results would remain the same. Moreover, we do not deal with markets with more than one risky asset. It does not seem likely that one could get results as explicit as we get for the case of one stock only (although Broadie et al. (1998) get explicit results for the multi-dimensional case, when volatility is constant). Nevertheless, the viscosity supersolution characterization of the minimal superreplication price would remain the same. (A related viscosity characterization is obtained in Buckdahn and $\mathrm{Hu}$ (1998) in a different context.)

Consider an economic agent, endowed with an initial capital $x$, who invests at each time $t \in[0, T]$ a proportion $\pi(t)$ of his wealth in the risky asset and the remaining wealth in the bank account. Here $\pi=\{\pi(t), 0 \leq t \leq T\}$ is an $\{\mathcal{F}(t)\}$ progressively measurable process with $\int_{0}^{T} \pi(t)^{2}\left(\sigma_{1}^{2}+\sigma_{2}^{2}\right)(t, S(t), Y(t)) \mathrm{d} t<\infty$, almost surely. Then the wealth process $X^{x, \pi}(t)$ satisfies the linear stochastic differential equation (for $t<T$ ),

$$
\begin{aligned}
\mathrm{d} X^{x, \pi}(t) & =\pi(t) X^{x, \pi}(t) \frac{\mathrm{d} S(t)}{S(t)}, \\
X^{x, \pi}(0) & =x .
\end{aligned}
$$

Let $K=[l, u],-\infty \leq l \leq 0 \leq u \leq \infty$ be an arbitrary closed convex set in $\mathbb{R}$ containing 0 . We say that a portfolio $\pi$ is $K$-admissible if

$$
\pi(t) \in K, \quad 0 \leq t \leq T
$$

holds $P$-a.s. The set of $K$-admissible portfolios will be denoted by $\mathcal{A}_{K}$.

Remark 2.2. The set $K$ specifies the constraints on borrowing and short-selling that our agent must adhere to. He/she cannot borrow more than $u$ times the amount he/she owns and cannot sell short more than $-l$ times his/her current wealth. Notice also that since the wealth process $\left\{X^{x, \pi}(t), 0 \leq t \leq T\right\}$ is a continuous process, it is clear from (2.5) that, given an initial wealth $x>0$, we have $X^{x, \pi}(t) \geq 0$ for all $t \in[0, T]$, almost surely. Therefore, in this model where the portfolio is defined as proportions of the wealth process, the no-bankruptcy condition is automatically satisfied. 
In this paper, we consider European contingent claims defined by a terminal payoff $g(S(T))$, where $g$ is a non-zero and non-negative function. Given such a contingent claim, we then consider the infimum $U(0)$ of initial capitals $x$, which induce a wealth process $X^{x, \pi}$ through some admissible portfolio $\pi \in \mathcal{A}_{K}$ such that $X^{x, \pi}$ hedges $g(S(T))$, i.e.

$$
U(0)=\inf \left\{x>0: \exists \pi \in \mathcal{A}_{K}, X^{x, \pi}(T) \geq g(S(T)) P \text {-a.s. }\right\} .
$$

It should be pointed out that we could also allow consumption (withdrawal of funds) in the above definitions, and the results would not change.

The main result of this paper is an explicit solution to the problem of calculating $U(0)$, as in Broadie et al. (1998), who deal with the Black-Scholes model with constraints.

\section{Shadow prices}

We introduce in this section the shadow state-price densities relevant to our incomplete market, following Cvitanić and Karatzas (1993) or Karatzas and Kou (1996). We denote by $\Lambda^{*}$ the set of all adapted, $\mathbb{R}$-valued processes $\lambda$. An appropriately defined subset of $\Lambda^{*}$ will correspond to the shadow prices associated with the incompleteness of the market due to the stochastic volatility. We also introduce the shadow prices relevant to the incompleteness of the market induced by the constraints: consider the support function of the convex set $-K$,

$$
\delta(z)=\sup _{x \in[l, u]}\{-x z\}=u z^{-}-l z^{+},
$$

and the associated effective domain

$$
\begin{aligned}
\tilde{K} & =\{z \in \mathbb{R}: \delta(z)<\infty\} \\
& =\{z \in \mathbb{R}: z \geq 0 \text { if } u=\infty \text { and } z \leq 0 \text { if } l=-\infty\} .
\end{aligned}
$$

Notice that $\delta \geq 0$ on $\tilde{K}$ since $0 \in[l, u]$. Then, let $\mathscr{D}^{*}$ be the set of all $\{\mathcal{F}(t)\}$ adapted processes $v=\{v(t), 0 \leq t \leq T\}$ such that $v(t) \in \tilde{K}$ for all $0 \leq t \leq T P$-a.s. Given $\lambda \in \Lambda^{*}$ and $v \in D^{*}$, we introduce the shadow state-price density process $M^{\lambda, \nu}$ by

$$
\begin{aligned}
\frac{\mathrm{d} M^{\lambda, v}(t)}{M^{\lambda, v}(t)} & =-\left(\frac{\mu-\lambda\left(\sigma_{2} / \gamma\right)+v}{\sigma_{1}}\right) \mathrm{d} W_{1}(t)-\frac{\lambda}{\gamma} \mathrm{d} W_{2}(t) \\
M^{\lambda, v}(0) & =1
\end{aligned}
$$

provided that the stochastic integrals can be defined. This happens to be too large a class of shadow state-price densities, and we will find it convenient to reduce it by considering subsets $\Lambda$ and $\mathscr{D}$ of $\Lambda^{*}$ and $\mathscr{D}^{*}$ (respectively), consisting only of almost surely bounded processes. The following standing assumption is a natural generalization of the standard assumption that the risk-neutral shadow state-price density is a martingale:

Assumption 3.1. The coefficient functions $\mu, \sigma_{1}, \sigma_{2}, \eta, \gamma$ defining the dynamics of $(S, Y)$ are such that

$$
\int_{0}^{T}\left[\left(\frac{\lambda}{\gamma}\right)^{2}+\left(\frac{\mu-\lambda\left(\sigma_{2} / \gamma\right)+v}{\sigma_{1}}\right)^{2}\right] \mathrm{d} u<\infty, \text { a.s. }
$$

and $M^{\lambda, v}$ are martingales, for all $(\lambda, v) \in \Lambda \times \mathscr{D}$. 
For example, the latter will be satisfied if the Novikov condition (see Karatzas and Shreve (1991)) is satisfied for all $M^{\lambda, \nu}$, with $\lambda$ and $v$ bounded almost surely.

Therefore, given any $\lambda \in \Lambda$ and $v \in \mathcal{D}$, we can define a probability measure $Q^{\lambda, v}$ equivalent to $P$, by $\mathrm{d} Q^{\lambda, v}=M^{\lambda, v}(T) \mathrm{d} P$. Then, by Girsanov's theorem,

$$
\begin{aligned}
& W_{1}^{\lambda, v}(t)=W_{1}(t)+\int_{0}^{t} \frac{\mu-\lambda\left(\sigma_{2} / \gamma\right)+v}{\sigma_{1}}(u) \mathrm{d} u, \\
& W_{2}^{\lambda, v}(t)=W_{2}(t)+\int_{0}^{t} \frac{\lambda}{\gamma}(u) \mathrm{d} u
\end{aligned}
$$

is a $Q^{\lambda, v}$ standard Brownian motion in $\mathbb{R}^{2}$ and the dynamics of the process $(S, Y)$ are driven by the SDE

$$
\begin{aligned}
& \frac{\mathrm{d} S(t)}{S(t)}=-v(t) \mathrm{d} t+\sigma_{1}(t, S(t), Y(t)) \mathrm{d} W_{1}^{\lambda, v}(t)+\sigma_{2}(t, S(t), Y(t)) \mathrm{d} W_{2}^{\lambda, v}(t) \\
& \mathrm{d} Y(t)=[\eta(t, S(t), Y(t))-\lambda(t)] \mathrm{d} t+\gamma(t, S(t), Y(t)) \mathrm{d} W_{2}^{\lambda, v}(t) .
\end{aligned}
$$

Therefore, under $Q^{\lambda, v}$ measure, the 'discounted' wealth process

$$
\tilde{X}(t):=X(t) \mathrm{e}^{-\int_{0}^{t} \delta(v(u)) \mathrm{d} u},
$$

corresponding to a strategy $\pi$ satisfies (suppressing the dependence on $t$ in coefficients)

$$
\mathrm{d} \tilde{X}(t)=\tilde{X}(t)\left[-(\pi v+\delta(v)) \mathrm{d} t+\pi \sigma_{1} \mathrm{~d} W_{1}^{\lambda, v}(t)+\pi \sigma_{2} \mathrm{~d} W_{2}^{\lambda, v}(t)\right] .
$$

Recalling the definition of $\delta$, we see that the discounted wealth process is a (non-negative) $Q^{\lambda, \nu}$-supermartingale. In particular, for a super-replicating process $X$ we obtain

$$
X(0) \geq E^{Q^{\lambda, v}}[\tilde{X}(T)] \geq E^{Q^{\lambda, v}}\left[g(S(T)) \exp \left(-\int_{0}^{T} \delta(v(u)) \mathrm{d} u\right)\right]
$$

A consequence of this is the next proposition.

Proposition 3.1. $U(0) \geq \sup _{(\lambda, v) \in \Lambda \times D} E^{Q^{\lambda, v}}\left[g(S(T)) \exp \left(-\int_{0}^{T} \delta(v(u)) \mathrm{d} u\right)\right]$.

Remark 3.1. A more general result of this kind, with equality, was obtained in Cvitanić and Karatzas (1993) and Karatzas and Kou (1996), in a (possibly) non-Markovian framework, but with somewhat stronger boundedness assumptions on the volatility coefficients. We will prove indirectly that the equality holds here also (under some assumptions), by characterizing the right-hand side in Proposition 3.1 quite explicitly, and showing that one can super-replicate $g(S(T))$ starting with the right-hand side as the initial investment.

From now on we also impose the following standing assumption.

Assumption 3.2. The payoff function $g(\cdot)$ is lower semicontinuous. 


\section{Necessary conditions from the Bellman equation}

As explained in the previous section, the super-replication cost at time $t$ has a lower bound $V\left(t, S_{t}, Y_{t}\right)$ given by the following control problem:

$$
V(t, s, y)=\sup _{(\lambda, v) \in \Lambda \times \mathbb{D}} E^{Q^{\lambda, v}}\left[g(S(T)) \exp \left(-\int_{t}^{T} \delta(v(u)) \mathrm{d} u\right) \mid(S(t), Y(t))=(s, y)\right] .
$$

In what follows, we assume that function $V(t, s, y)$ is finite. We have the following property of the function $V$ (we use the definition of Crandall, Ishii and Lions (1992) for viscosity supersolutions).

Proposition 4.1. The value function $V(t, s, y)$ is a lower semicontinuous viscosity supersolution to the Bellman equation,

$$
\inf _{(\lambda, v) \in \mathbb{R} \times \tilde{K}}\left[\mathcal{L} v+\mathcal{g} v+v s v_{s}+\lambda v_{y}+\delta(v) v\right]=0
$$

where

$$
\begin{aligned}
\mathcal{L} v & =-v_{t}-\frac{1}{2} s^{2} \sigma^{2} v_{s s} \text { with } \sigma^{2}=\sigma_{1}^{2}+\sigma_{2}^{2}, \\
g v & =-\eta v_{y}-\frac{1}{2} \gamma^{2} v_{y y}-s \sigma_{2} \gamma v_{s y} .
\end{aligned}
$$

Moreover,

$$
V(T-, s, y) \geq g(s)
$$

Proof. See Appendix A.

Notice that we only establish that $V$ is a supersolution to the Bellman equation. Our control problem is singular (due to the non-compactness of the set of controls), and it is well known that there are many examples where the Bellman equation then fails to hold. In general, that is also going to be the case in this paper. We could have used the normalized Bellman equation as in Krylov (1980), which involves stronger conditions on the model in order to define generalized derivatives of the value function $V$. The main advantage of the viscosity approach is that it requires weaker conditions on the regularity of the value function $V$. In fact, we will show that the characterization of the function $V$ as a lower semicontinuous viscosity supersolution of the Bellman equation is sufficient for our analysis.

We now derive some implications from Proposition 4.1 which will be sufficient to deduce the super-replication cost $U(0)$. Fix some $(t, s, y) \in[0, T) \times \mathbb{R}_{+}^{*} \times \mathbb{R}$. Then by the viscosity supersolution definition, for any function $\varphi \in C^{2}\left([0, T) \times \mathbb{R}_{+}^{*} \times \mathbb{R}\right)$ such that

$$
0=(V-\varphi)(t, s, y)=\min _{[0, T) \times \mathbb{R}_{+}^{*} \times \mathbb{R}}(V-\varphi),
$$

we have

$$
\left(\mathcal{L} \varphi+\mathcal{g} \varphi+v s \varphi_{s}+\lambda \varphi_{y}+\delta(\nu) \varphi\right)(t, s, y) \geq 0, \quad \text { for all }(\lambda, v) \in \mathbb{R} \times \tilde{K} .
$$

By sending $\lambda$ to $\pm \infty$ it becomes clear that we must have

$$
\varphi_{y}(t, s, y)=0
$$

for any test function $\varphi$ satisfying (4.6). We now need the following result. 
Lemma 4.1. Let $V: A \times B \rightarrow \mathbb{R}, A \subseteq \mathbb{R}^{m}, B \subseteq \mathbb{R}$, be a lower semicontinuous supersolution to the equation

$$
H\left(x, y, V_{y}, V_{y y}\right)=0, \quad x \in A, \quad y \in B,
$$

where $H: A \times B \times \mathbb{R}^{2} \rightarrow \mathbb{R}$ is a continuous function. Assume also that $V(\cdot, \cdot) \geq-C$ for some constant $C>0$. Then, for any fixed $x_{0} \in A$, the function $V\left(x_{0}, \cdot\right)$ is a lower semicontinuous supersolution to the equation

$$
H\left(x_{0}, y, V_{y}, V_{y y}\right)=0, \quad y \in B .
$$

Proof. Let $\phi: B \rightarrow \mathbb{R}$ be a $C^{2}$ test function such that, for some $y_{0} \in B$, we have

$$
0=V\left(x_{0}, y_{0}\right)-\phi\left(y_{0}\right)<V\left(x_{0}, y\right)-\phi(y), \quad \forall y \in B .
$$

Since it is sufficient to check the viscosity property on the strict minima of $V\left(x_{0}, \cdot\right)-\phi(\cdot)$, all we have to do is prove that

$$
H\left(x_{0}, y_{0}, \phi_{y}\left(y_{0}\right), \phi_{y y}\left(y_{0}\right)\right) \geq 0 \text {. }
$$

We define

$$
\psi^{(n)}(x, y):=\phi(y)-n\left|x-x_{0}\right|^{2}, \quad x \in A, \quad y \in B,
$$

for all $n \in \mathbb{N}$, and

$$
D^{(n)}(x, y):=V(x, y)-\psi^{(n)}(x, y) .
$$

Let $I$ be a compact neighborhood of $\left(x_{0}, y_{0}\right)$ in $A \times B$. Being lower semicontinous, $D^{(n)}$ attains its minimum on $I$, say at a point $\left(x_{n}, y_{n}\right)$. There then exists a pair $\left(x^{*}, y^{*}\right) \in I$ and a (relabeled) subsequence such that

$$
\left(x_{n}, y_{n}\right) \rightarrow\left(x^{*}, y^{*}\right) \in I .
$$

Now, since $V$ is bounded from below and $\phi$ is continuous, we see that if $x^{*} \neq x_{0}$, then $D^{(n)}\left(x_{n}, y_{n}\right) \rightarrow \infty$, as $n \rightarrow \infty$. This is a contradiction, since, for example, $D^{(n)}\left(x_{n}, y_{n}\right) \leq$ $D^{(n)}\left(x_{0}, y_{0}\right)=V\left(x_{0}, y_{0}\right)-\phi\left(y_{0}\right)$. We conclude that

$$
x^{*}=x_{0} .
$$

On the other hand, since $V\left(x_{0}, y_{0}\right)-\phi\left(y_{0}\right)=D^{(n)}\left(x_{0}, y_{0}\right) \geq D^{(n)}\left(x_{n}, y_{n}\right)$, we also have

$$
\begin{aligned}
V\left(x_{0}, y_{0}\right)-\phi\left(y_{0}\right) & \geq \liminf _{n} D^{(n)}\left(x_{n}, y_{n}\right) \\
& \geq V\left(x_{0}, y^{*}\right)-\phi\left(y^{*}\right),
\end{aligned}
$$

by lower semicontinuity. Consequently, (4.11) implies that

$$
y^{*}=y_{0} .
$$

Now, for large enough $n,\left(x_{n}, y_{n}\right)$ is a point of local minimum of $D^{(n)}$ on $I$ because it converges to $\left(x_{0}, y_{0}\right)$ (it is a classical local minimum if $\left(x_{0}, y_{0}\right)$ is in the interior of $A \times B$; otherwise, we can always extend our functions appropriately so that $\left(x_{0}, y_{0}\right)$ becomes an interior point, and so that the local minimality is preserved). Thus, by the viscosity property of $V(x, y)$, we have

$$
H\left(x_{n}, y_{n}, \psi_{y}^{(n)}\left(x_{n}, y_{n}\right), \psi_{y y}^{(n)}\left(x_{n}, y_{n}\right)\right) \geq 0 .
$$

Sending $n \rightarrow \infty$ we get (4.12), and we have completed the proof.

From this we get the following result. 


\section{Lemma 4.2. The function $V$ does not depend on $y$.}

Proof. From (4.8) we conclude that $V(t, s, y)$ is a lower semicontinuous viscosity supersolution to the equation $v_{y}=0$. By Lemma 4.1, we also have that the function $V(t, s, \cdot)$ is a viscosity supersolution of the same equation, for any fixed $(t, s)$. We then fix a pair $(t, s)$ and omit $t, s$ in the following. Fix also $y_{0}, y_{2}$ and consider $y_{0}<y_{1}<y_{2}$ and a test function $\varphi$ such that

$$
(V-\varphi)\left(y_{1}\right)=0=\min _{y_{0} \leq y \leq y_{2}}(V-\varphi)(y) .
$$

Since the viscosity property remains the same if we consider local minima rather than global minima, we conclude that

$$
\varphi_{y}\left(y_{1}\right)=0 .
$$

Think of $y$ as a time variable, and the function $V$ as a viscosity supersolution to the parabolic PDE

$$
v_{y}=0, \quad y \in\left[y_{0}, y_{2}\right], \quad v\left(y_{2}\right)=V\left(y_{2}\right) .
$$

Since the constant function $v=V\left(y_{2}\right)$ is also a solution, we have, by the maximum principle (see Crandall et al. (1992), Theorems 3.3 and 8.2; notice also that we reverse the direction of the time variable in this proof):

$$
V(y) \geq V\left(y_{2}\right), \quad y_{0} \leq y \leq y_{2} .
$$

Since $y_{0}, y_{2}$ are arbitrary, $V$ is non-increasing. To prove the opposite inequality, define

$$
W(y)=V\left(y_{2}+y_{0}-y\right), \quad y_{0} \leq y \leq y_{2} .
$$

Fix some $y_{1} \in\left(y_{0}, y_{2}\right)$ and consider a $C^{1}$ test function $\psi$ such that

$$
(W-\psi)\left(y_{1}\right)=\min _{y_{0} \leq y \leq y_{2}}(W-\psi)(y)
$$

Then, defining the $C^{1}$ test function $\varphi$ by

$$
\varphi(y)=\psi\left(y_{2}+y_{0}-y\right), \quad y_{0} \leq y \leq y_{2},
$$

we see that

$$
(V-\varphi)\left(y_{2}+y_{0}-y_{1}\right)=\min _{y_{0} \leq y \leq y_{2}}(V-\varphi)(y)
$$

by an obvious change of variable. Therefore, we must have

$$
\psi_{y}\left(y_{2}+y_{0}-y_{1}\right)=-\varphi_{y}\left(y_{1}\right)=0 \text {. }
$$

It follows that $W$ is a supersolution to the parabolic PDE

$$
v_{y}=0, \quad y \in\left[y_{0}, y_{2}\right], \quad v\left(y_{2}\right)=V\left(y_{0}\right) .
$$

By the above argument this means that $W(y) \geq W\left(y_{2}\right)$, or $V(y) \geq V\left(y_{0}\right)$ for $y \in\left[y_{0}, y_{2}\right]$. Since $y_{0}, y_{2}$ are arbitrary, $V$ is non-decreasing, hence constant (in $y$ ). 
Accordingly, in the following, we omit the argument $y$ in the function $V$. It then follows from (4.7) that, in a viscosity sense,

$$
\mathcal{L} V+v s V_{s}+\delta(v) V \geq 0 \text { for all } v \in \tilde{K}
$$

Writing condition (4.13) for $v=0 \in \tilde{K}$, we get

$$
\mathcal{L} V(t, s) \geq 0, \quad(t, s) \in[0, T) \times \mathbb{R}_{+}^{*} .
$$

Next, suppose that there exists some $v_{0} \in \tilde{K}$ and $\left(t_{0}, s_{0}\right) \in[0, T) \times \mathbb{R}_{+}^{*}$ such that $\left[v_{0} s V_{s}+\right.$ $\left.\delta\left(v_{0}\right) V\right]\left(t_{0}, s_{0}\right)<0$. Then by the cone property of $\tilde{K}$, the left-hand side of (4.13) can be sent to $-\infty$, which is a contradiction. Therefore, we must have, in a viscosity sense,

$$
\left(v s V_{s}+\delta(v) V\right)(t, s) \geq 0, \quad \text { for all } v \in \tilde{K} \text { and }(t, s) \in[0, T) \times \mathbb{R}_{+}^{*} .
$$

Combining (4.14) and (4.15), we get the following proposition.

Proposition 4.2. The function $V$ does not depend on $y$ and is a viscosity supersolution to the equation

$$
\min \left\{\mathcal{L} v, \inf _{v \in \tilde{K}}\left(v s v_{s}+\delta(v) v\right)\right\}=0 .
$$

In the following sections, we use this proposition in order to characterize explicitly the solution $U(0)$ to the hedging problem under different assumptions on the volatility function.

\section{Unbounded volatility}

In this section we provide an explicit solution to the hedging problem under the following conditions.

Assumption 5.1. $\sup _{y \in \mathbb{R}} \sigma(t, s, y)=\infty$ for all $(t, s) \in[0, T) \times \mathbb{R}_{+}^{*}$.

Assumption 5.2. $\inf _{y \in \mathbb{R}} \sigma(t, s, y)=0$ for all $(t, s) \in[0, T) \times \mathbb{R}_{+}^{*}$.

Then we have the following preliminary result on the function $V$.

Lemma 5.1. (i) Under Assumption 5.1, the function $V$ is concave in sor any fixed $t \in[0, T)$. (ii) Under Assumption 5.2, the function $V$ is non-increasing in t for any fixed $s \in \mathbb{R}_{+}^{*}$.

Proof. From equation (4.14), we know that $V$ is a viscosity supersolution to

$$
-v_{t}(t, s)-\frac{1}{2} \sigma^{2}(t, s, y) s^{2} v_{s s}(t, s)=0, \quad(t, s, y) \in[0, T) \times \mathbb{R}_{+}^{*} \times \mathbb{R} .
$$

(i) By considering a maximizing sequence of $\sigma(t, s, y)$ for any fixed $(t, s) \in[0, T) \times \mathbb{R}_{+}^{*}$, we see that the function $V(\cdot, \cdot)$ is a lower semicontinuous viscosity supersolution of the equation $-v_{s s}=0$. By Lemma 4.1, the function $V(t, \cdot)$ is a supersolution to the same equation, for any fixed $t$. We fix $t$ and suppress the dependence on $t$ in the following discussion. Since $V$ is non-negative, it can easily be checked that $V$ is also a viscosity supersolution of the equation $\varepsilon v-v_{s s}=0$ for all $\varepsilon>0$. Let $[a, b]$ be any closed interval in $\mathbb{R}_{+}^{*}$ and consider the differential equation

$$
\left(\varepsilon v-v_{s S}\right)(s)=0, \quad v(a)=V(a) \text { and } v(b)=V(b) .
$$


Then, since $V(\cdot)$ is a lower semicontinuous viscosity supersolution of the last equation, by the maximum principle (see Crandall et al. (1992), Theorems 3.3 and 8.2), we see that

$$
V(s) \geq \frac{V(a)\left[\mathrm{e}^{\sqrt{\varepsilon}(b-s)}-1\right]+V(b)\left[\mathrm{e}^{\sqrt{\varepsilon}(s-a)}-1\right]}{\mathrm{e}^{\sqrt{\varepsilon}(b-a)}-1},
$$

for all $\varepsilon>0$, since the function on the right-hand side is a solution to the same equation. Sending $\varepsilon$ to zero in the last inequality gives

$$
V(s) \geq[V(b)-V(a)] \frac{b-s}{b-a}+V(a),
$$

for all $s \in[a, b]$. Applying the last inequality to $s=\lambda a+(1-\lambda) b$ for some $\lambda \in[0,1]$ gives

$$
V(\lambda a+(1-\lambda) b) \geq \lambda V(a)+(1-\lambda) V(b),
$$

for all $a, b \in \mathbb{R}_{+}^{*}$.

(ii) By considering a minimizing sequence of $\sigma(t, s, y)$ for any fixed $(t, s) \in[0, T) \times \mathbb{R}_{+}^{*}$, we see that the function $V(\cdot, \cdot)$ is a lower semicontinuous viscosity supersolution of the equation $-v_{t}=0$. The result follows as in part (i) by an application of Lemma 4.1 and the maximum principle.

Now, let $\mathscr{H}$ be the set of all functions mapping $\mathbb{R}_{+}^{*}$ into $\mathbb{R} \cup\{\infty\}$. As in Broadie et al. (1998), we introduce the operator $H$ defined on $\mathscr{H}$ by

$$
H(h)(s)=\hat{h}(s)=\sup _{v \in \tilde{K}} h\left(s \mathrm{e}^{-v}\right) \mathrm{e}^{-\delta(v)}, \quad s \in \mathbb{R}_{+}^{*} .
$$

Then we have the following result on function $V$.

Lemma 5.2. For any $t \in[0, T)$, the function $V(t, \cdot)$ is invariant under $H$, that is

$$
\hat{V}(t, \cdot)=V(t, \cdot), \quad t \in[0, T) .
$$

Proof. From equation (4.16), we have that for all $v \in \tilde{K}, V(t, \cdot)$ is a lower semicontinuous viscosity supersolution of

$$
v s v_{s}(t, s)+\delta(v) v(t, s)=0, \quad v\left(t, s_{0}\right)=V\left(t, s_{0}\right) .
$$

Since $\delta$ is non-negative, it follows from the maximum principle in the viscosity sense (see Crandall et al. (1992)) that

$$
V(t, s) \geq V\left(t, s_{0}\right)\left(\frac{s}{s_{0}}\right)^{-\delta(v) / v}, \quad v \in \tilde{K},
$$

since the right-hand side solves the same PDE. By taking $s_{0}=s \mathrm{e}^{-v}$, we see that

$$
V(t, s) \geq V\left(t, s \mathrm{e}^{-v}\right) \mathrm{e}^{-\delta(v)}, \quad v \in \tilde{K}
$$

which proves that $V(t, \cdot) \geq \hat{V}(t, \cdot)$ for all $t \in[0, T)$. The reverse inequality is trivial since $0 \in \tilde{K}$. 
Next, we consider the following optimal stopping problem

$$
\tilde{g}(s)=\sup _{\tau \in \mathcal{T}} E\left[\hat{g}\left(Z^{s}(\tau)\right)\right], \quad s \in \mathbb{R}_{+}^{*},
$$

where $\mathcal{T}$ is the set of all non-negative stopping times and $\mathrm{d} Z^{s}(t)=Z^{s}(t) \mathrm{d} W(t), Z^{s}(0)=s$. We provide the following characterizations of the functions $\hat{g}$ and $\tilde{g}$.

Lemma 5.3. $\hat{g}$ is the smallest function invariant under $H$ such that $\hat{g} \geq g$.

Proof. (i) From the definition of the operator $H$, it is clear that $\hat{g} \geq g$, since $0 \in \tilde{K}$, and $H(\hat{g})=\hat{g}$.

(ii) Let $h$ be any function invariant under $H$ such that $h \geq g$. Then we have

$$
h(s)=\sup _{v \in \tilde{K}} h\left(s \mathrm{e}^{-v}\right) \mathrm{e}^{-\delta(v)} \geq \sup _{v \in \tilde{K}} g\left(s \mathrm{e}^{-v}\right) \mathrm{e}^{-\delta(v)}=\hat{g}(s)
$$

which ends the proof.

Lemma 5.4. $\tilde{g}$ is the smallest concave function such that $\tilde{g} \geq g$ and $H(\tilde{g})=\tilde{g}$.

Proof. (i) From the definition of $\tilde{g}$ in (5.2), $\tilde{g}$ is a superharmonic function, hence concave.

(ii) It is also clear that $\tilde{g} \geq g$.

(iii) We now prove that $H(\tilde{g})=\tilde{g}$. Since $Z^{s \mathrm{e}^{-v}}(t)=\mathrm{e}^{-v} Z^{s}(t)$, we have for all $v \in \tilde{K}$

$$
\tilde{g}\left(s \mathrm{e}^{-v}\right) \mathrm{e}^{-\delta(v)}=\sup _{\tau \in \mathcal{T}} E\left[\hat{g}\left(Z^{s}(\tau) \mathrm{e}^{-v}\right) \mathrm{e}^{-\delta(v)}\right] .
$$

This implies that

$$
H(\tilde{g})(s)=\sup _{v \in \tilde{K}} \sup _{\tau \in \mathcal{T}} E\left[\hat{g}\left(Z^{s}(\tau) \mathrm{e}^{-v}\right) \mathrm{e}^{-\delta(v)}\right] .
$$

By taking $v=0$ in the right-hand side of (5.3), we obviously have $H(\tilde{g}) \geq \tilde{g}$. By applying Jensen's inequality to the right-hand side of (5.3), we obtain

$$
\begin{aligned}
H(\tilde{g})(s) & \leq \sup _{\tau \in \mathcal{T}} E\left[\sup _{v \in \tilde{K}} \hat{g}\left(Z^{s}(\tau) \mathrm{e}^{-v}\right) \mathrm{e}^{-\delta(v)}\right] \\
& =\sup _{\tau \in \mathcal{T}} E\left[H(\hat{g})\left(Z^{s}(\tau)\right)\right] .
\end{aligned}
$$

Since $H(\hat{g})=\hat{g}$, (5.4) implies that $H(\tilde{g}) \leq \tilde{g}$ and finally $H(\tilde{g})=\tilde{g}$.

(iv) Let $h$ be a concave function such that $h \geq g$ and $H(h)=h$. By Lemma 5.3, $h \geq \hat{g}$, and so

$$
E\left[h\left(Z^{s}(\tau)\right)\right] \geq E\left[\hat{g}\left(Z^{s}(\tau)\right)\right],
$$

for all $s>0$ and all stopping times $\tau \in \mathcal{T}$. By Jensen's inequality and since $Z^{s}$ is a martingale, we have, for all $n \in \mathbb{N}$,

$$
h(s) \geq E\left[\hat{g}\left(Z^{s}(\tau)\right)\right]
$$

for all stopping times $\tau \leq n$. This implies that

$$
h(s) \geq \sup _{\tau \leq n} E\left[\hat{g}\left(Z^{s}(\tau)\right)\right],
$$

and finally by sending $n$ to infinity, that $h \geq \tilde{g}$. 
Lemma 5.5. For all $s \in \mathbb{R}_{+}^{*}$, we have

$$
\tilde{g}(s)=\inf \left\{c>0: \exists \Delta \in \mathbb{R}, s \Delta / c \in K, \text { and } \forall z \in \mathbb{R}_{+}^{*}, c+\Delta(z-s) \geq \hat{g}(z)\right\} .
$$

Proof. Let us define the function $f$ by

$$
f(s)=\inf \left\{c>0: \exists \Delta \in \mathbb{R}, s \Delta / c \in K \text {, and } \forall z \in \mathbb{R}_{+}^{*}, c+\Delta(z-s) \geq \hat{g}(z)\right\} .
$$

Since it is defined as the infimum of affine functions, $f$ is a concave function. It is also clear that $f \geq \hat{g} \geq g$. Let us prove that $\hat{f}=f$ or equivalently that $\hat{f} \leq f$ since the reverse inequality is always true. By definition of $f$, we have for all $v \in \tilde{K}$

$$
\begin{aligned}
f\left(s \mathrm{e}^{-v}\right) \mathrm{e}^{-\delta(v)}= & \inf \left\{c \mathrm{e}^{-\delta(v)}>0: \exists \Delta \in \mathbb{R}, s \mathrm{e}^{-v} \Delta / c \in K,\right. \\
& \text { and } \left.\forall z \in \mathbb{R}_{+}^{*}, c+\Delta\left(z-s e^{-v}\right) \geq \hat{g}(z)\right\} \\
= & \inf \left\{c^{\prime}>0: \exists \Delta^{\prime} \in \mathbb{R}, s \Delta^{\prime} / c^{\prime} \in K,\right. \\
& \text { and } \left.\forall z^{\prime} \in \mathbb{R}_{+}^{*}, c^{\prime}+\Delta^{\prime}\left(z^{\prime}-s\right) \geq \hat{g}\left(z^{\prime} \mathrm{e}^{-v}\right) \mathrm{e}^{-\delta(v)}\right\} .
\end{aligned}
$$

Taking the supremum of this last relation over $v \in \tilde{K}$, we obtain

$$
\hat{f}(s) \leq \inf \left\{c>0: \exists \Delta \in \mathbb{R}, s \Delta / c \in K, \forall z \in \mathbb{R}_{+}^{*}, c+\Delta(z-s) \geq \sup _{v \in \tilde{K}} \hat{g}\left(z \mathrm{e}^{-v}\right) \mathrm{e}^{-\delta(v)}\right\} .
$$

Since $H(\hat{g})=\hat{g}$, the last inequality means that $\hat{f} \leq f$ and so $\hat{f}=f$. Let $h$ be a concave function such that $h \geq g$ and $\hat{h}=h$ for all $s>0$. By Lemma 5.3, $h \geq \hat{g}$. Since $h$ is concave, we have, for all $s, z \in \mathbb{R}_{+}^{*}$,

$$
h(s)+h_{-}^{\prime}(s)(z-s) \geq h(z) \geq \hat{g}(z),
$$

where $h_{-}^{\prime}$ is the left derivative of the concave function $h$. Since $g$ is a non-zero and nonnegative function, so is $h$; moreover, since $h$ is concave, it is in fact positive on $\mathbb{R}_{+}^{*}$. Let us now prove that $\Delta=h_{-}^{\prime}(s)$ satisfies $s \Delta / h(s) \in K$. Since $\hat{h} \leq h$, we have $h(s) \geq h\left(s \mathrm{e}^{-v}\right) \mathrm{e}^{-\delta(v)}$ and therefore

$$
\log h(s)-\log h\left(s \mathrm{e}^{-v}\right) \geq-\delta(v), \quad s>0 \text { and } v \in \tilde{K} .
$$

Since $\tilde{K}$ is a cone and $\delta$ is positively homogeneous, this implies that for any $s>0$ and $v \in$ $\tilde{K} \cap \mathbb{R}_{+}^{*}$,

$$
\frac{\log h(s)-\log h\left(s \mathrm{e}^{-\varepsilon v}\right)}{s\left(1-\mathrm{e}^{-\varepsilon v}\right)} \geq \frac{-\varepsilon \delta(v)}{s\left(1-\mathrm{e}^{-\varepsilon v}\right)}, \quad \varepsilon>0,
$$

and by sending $\varepsilon$ to zero, we get

$$
v \frac{s h_{-}^{\prime}(s)}{h(s)}+\delta(v) \geq 0, \quad v \in \tilde{K} \cap \mathbb{R}_{+}
$$

By a similar argument (using the change of variable $s^{\prime}=s \mathrm{e}^{-v}$ ), one shows that the last inequality also holds for $v \in \tilde{K} \cap \mathbb{R}_{-}$. Then, by Theorem 13.1 of Rockafellar (1970), this 
proves that $s \Delta / h(s) \in K$. Therefore, it follows by the definition of $f$ that $h \geq f$. By Lemma 5.4, we conclude that $f=\tilde{g}$.

From the last lemma, the amount $\tilde{g}(S(0))$ is the infimum of initial capitals of buy-and-hold strategies which dominate the contingent claim defined by the payoff function $\hat{g}$. It is easily checked that the infimum is attained and that the associated portfolio can be taken as

$$
\tilde{\pi}(t)=\frac{\tilde{g}_{-}^{\prime}(S(0)) S(t)}{\tilde{g}(S(0))+\tilde{g}_{-}^{\prime}(S(0))[S(t)-S(0)]},
$$

where the denominator of the right-hand side term of the last equation is equal to $X^{\tilde{g}(S(0)), \tilde{\pi}}(t)$, the wealth at time $t$ associated with the strategy $\tilde{\pi}$ and the initial capital $\tilde{g}(S(0))$. Notice that, since $\tilde{g}$ is concave and dominates the non-zero and non-negative function $g$, we have $X^{\tilde{g}(S(0)), \tilde{\pi}}(t)>0$ and

$$
\tilde{\pi}(t) \geq 0, \quad 0 \leq t \leq T .
$$

Furthermore, as in the proof of Lemma 5.5, we see that the portfolio strategy $\tilde{\pi}$ is $K$-admissible at time zero, i.e. $\tilde{\pi}(0) \in K$.

Remark 5.1. In the particular case $\tilde{g}_{-}^{\prime}(S(0))=0$, it is clear that $\tilde{\pi}=0$ and therefore the portfolio strategy $\tilde{\pi}$ is $K$-admissible.

In the general case, we have the following result.

Lemma 5.6. Suppose $\tilde{\pi}(0) \neq 0$. Then the portfolio strategy $\tilde{\pi}$ is $K$-admissible if and only if $u \geq 1$.

Proof. From (5.5), we have $\tilde{\pi}(t) \geq 0 \geq l$ for all $t \in[0, T]$. Therefore, the portfolio strategy $\tilde{\pi}$ is $K$-admissible if and only if

$$
\frac{\Delta z}{c+\Delta(z-s)} \leq u, \quad \text { for all } z>0
$$

where $s=S(0), c=\tilde{g}(S(0))$ and $\Delta=\tilde{g}_{-}^{\prime}(S(0)) \geq 0$. Since $\Delta$ is assumed to be non-zero, this can be written equivalently as

$$
z(1-u) \leq \frac{u}{\Delta}(c-\Delta s), \quad \text { for all } z>0 .
$$

Now, by the concavity of $\tilde{g}$, we have $c+\Delta(z-s) \geq \tilde{g}(z) \geq 0$ for all $z>0$. By sending $z$ to zero, we see that the right-hand side of (5.6) is non-negative. Therefore (5.6) holds if and only if $u \geq 1$.

Remark 5.2. The restriction $u \geq 1$ means that any admissible portfolio strategy $\pi$ is such that the corresponding wealth proportion invested in the non-risky asset satisfies $1-\pi(t) \geq 1-u$ with $1-u \leq 0$. In other words, we can keep all the money in the stock, if we want to.

Remark 5.3. In the case $u \geq 1$, we have shown that $\tilde{g}(s)$ is the minimal initial capital required in order to perform a $K$-admissible buy-and-hold strategy which allows us to dominate the contingent claim defined by the payoff function $\hat{g}$. The last assertion always holds (without 
assuming $u \geq 1$ ) whenever $\tilde{g}_{-}^{\prime}(S(0))=0$ (see Remark 5.1). Therefore, by the definition of $U$ (see Section 2), since $\hat{g} \geq g$, it follows that

$$
U(0) \leq \tilde{g}(S(0))
$$

whenever $u \geq 1$ or $\tilde{g}_{-}^{\prime}(S(0))=0$.

We now state the main result of this section which holds for lower semicontinuous, nonnegative and non-zero payoff functions $g$, and under the assumptions of Sections 2 and 3.

Theorem 5.1. Let Assumptions 5.1 and 5.2 hold and suppose that

$$
\text { either } \tilde{g}_{-}^{\prime}(S(0))=0 \quad \text { or } \quad u \geq 1 \text {. }
$$

Then we have

$$
U(0)=\tilde{g}(S(0)) .
$$

Proof. From Lemma 5.1(i), the function $s \mapsto V(t, s)$ is concave for all $0 \leq t<T$. Moreover, from (4.5) and Lemma 5.1(ii), we have

$$
V(t, s) \geq g(s), \quad(t, s) \in[0, T) \times \mathbb{R}_{+}^{*} .
$$

Furthermore, from Lemma 5.2, $V(t, \cdot)$ is invariant under $H$ for all $t \in[0, T)$. From Lemma 5.4, this implies that $V(t, s) \geq \tilde{g}(s)$ for all $(t, s) \in[0, T) \times \mathbb{R}_{+}^{*}$. Therefore, from Proposition 3.1, we have

$$
U(0) \geq \tilde{g}(S(0)) .
$$

The required result follows from (5.7).

The conditions of Theorem 5.1 can be weakened for convex payoff functions $g$.

Theorem 5.2. Suppose $g$ is convex and let Assumption 5.1 and the restriction (5.8) hold. Then we have

$$
U(0)=\tilde{g}(S(0)) .
$$

Proof. The inequality $U(0) \leq \tilde{g}(S(0))$ follows from (5.7). To see that the reverse inequality holds, notice that by considering the controls $(\lambda, v)=0,(4.1)$ implies that

$$
\begin{aligned}
V(t, s) & \geq E^{Q^{0,0}}[g(S(T)) \mid(S(t), Y(t))=(s, y)] \\
& \geq g(s), \quad(t, s) \in[0, T) \times \mathbb{R}_{+}^{*},
\end{aligned}
$$

where we have used Jensen's inequality and the martingale property of $\{S(t), 0 \leq t \leq T\}$ under $Q^{0,0}$. Now, from Lemma 5.2, $V(t, \cdot)$ is invariant under $H$ for all $t \in[0, T)$ and, by Lemma 5.1(i), $V$ is concave. Therefore, the required inequality follows from Lemma 5.4 and Proposition 3.1.

Remark 5.4. From the proof of Theorems 5.1 and 5.2, it is clear that without the restriction (5.8), we still have

$$
U(0) \geq \tilde{g}(S(0)) .
$$

Remark 5.5. It is now clear that the HJB equation (4.2) for $V$ is not necessarily satisfied as an equality. In particular, this is the case if the concave envelope $\tilde{g}(\cdot)$ is strictly concave. 


\section{Bounded volatility}

Assume first that there are no constraints.

Assumption 6.1. (Bounded volatility..)

$$
\inf _{y \in \mathbb{R}} \sigma(t, s, y)=\underline{\sigma}(t, s) \text { and } \sup _{y \in \mathbb{R}} \sigma(t, s, y)=\bar{\sigma}(t, s), \quad \text { for all }(t, s) \in[0, T) \times \mathbb{R}_{+}^{*},
$$

where $\underline{\sigma}$ and $\bar{\sigma}$ satisfy the same assumptions as $\sigma$.

This is the framework of Avellaneda et al. (1995), and we first want to show that we can recover their result that the minimal super-replication price in this market satisfies $U(0) \leq \tilde{V}(0, S(0))$, where $\tilde{V}(t, s)$ is a solution to the Barenblatt PDE

$$
\begin{aligned}
-v_{t}+\frac{1}{2} s^{2} \underline{\sigma}^{2}\left(v_{s s}\right)^{-}-\frac{1}{2} s^{2} \bar{\sigma}^{2}\left(v_{s s}\right)^{+} & =0, \\
v(T, s) & =g(s),
\end{aligned}
$$

assuming that a unique classical solution exists. (In Appendix B, we provide sufficient conditions on the coefficients $\underline{\sigma}, \bar{\sigma}$ and the terminal payoff function $g$, which ensure the existence of a unique solution to the Barenblatt PDE (6.1).

In fact, we shall prove here that $U(0)=\tilde{V}(0, S(0))$. Let $\tilde{V}$ be the solution to (6.1)-(6.2). We first show that $V \geq \tilde{V}$, hence $U(0) \geq \tilde{V}(0, S(0))$. We already know that $V$ does not depend on $y$. Moreover, from (4.14) we conclude that $V$ is a lower semicontinuous viscosity supersolution of $(6.1)$, with $V(T-, s) \geq g(s)$ for all $s>0$. Therefore, the maximum principle ensures that $V \geq \tilde{V}$.

The opposite inequality $\tilde{V}(0, S(0)) \geq U(0)$ is quite straightforward. It is sufficient to prove that one can super-replicate $g(S(T))$ if one starts with the initial capital $\tilde{V}(0, S(0))$ at time 0 . From (6.1), it is easily seen that $-\mathscr{L} \tilde{V} \leq 0$. By Itô's rule we then get

$$
\begin{aligned}
g(S(T)) & =\tilde{V}(T, S(T)) \\
& \leq \tilde{V}(0, S(0))+\int_{0}^{T} \tilde{V}_{S}(t, S(t)) S(t)\left[\sigma_{1} \mathrm{~d} W_{1}^{0}(t)+\sigma_{2} \mathrm{~d} W_{2}^{0}(t)\right] .
\end{aligned}
$$

However, the right-hand side is the value at $T$ of a wealth process starting with $\tilde{V}(0, S(0))$ at time 0 , holding $\tilde{V}_{S}(t, S(t))$ shares of stock at time $t$, and it super-replicates $g(S(T))$.

Remark 6.1. The proof of $V \geq \tilde{V}$ given above remains the same when we assume that $\tilde{V}$ is only a viscosity solution to the Barenblatt PDE.

We now extend the previous result to the case of constraints, given linear dynamics for $S$.

Assumption 6.2. Functions $\eta(t, s, y), \gamma(t, s, y)$ and $\sigma_{i}(t, s, y), i=1,2$, do not depend on $s$.

The following result holds for lower semicontinuous, non-negative and non-zero payoff functions $g$ and under the assumptions of Sections 2 and 3.

Theorem 6.1. Given Assumptions 6.1 and 6.2, and in the presence of constraints described by the set $K=[l, u]$, the minimal super-replicating cost is given by $U(0)=\tilde{V}(0, S(0))$, where $\tilde{V}$ is the unique classical solution (if one exists) to the Barenblatt PDE (6.1), with the terminal condition

$$
\tilde{V}(T, s)=\hat{g}(s)
$$


where $\hat{g}=H(g)$ is defined in (5.1). The corresponding super-replicating portfolio is given by

$$
\pi(t)=\frac{S(t) \tilde{V}_{s}(t, S(t))}{\tilde{V}(t, S(t))} .
$$

Moreover, if there are no constraints $(K=\mathbb{R})$, the same is valid without using Assumption 6.2.

Proof. We have already proved the last statement in the theorem. For the rest, we will use representation (4.1) for $V(t, s)$. We follow the probabilistic argument of Broadie et al. (1998). Write, for a fixed $v, S^{0}(u)=S(u) \exp \left(\int_{t}^{u} v(s) \mathrm{d} s\right)$ for $t \leq u \leq T$ and notice that the distribution of $\left\{S^{0}(u), t \leq u \leq T\right\}$ under $Q^{\lambda, v}$ does not depend on $v$. Then, by Jensen's inequality and the convexity of $\delta$, and using the fact that for $v(\cdot) \in \mathscr{D}$ we have $\int_{t}^{T} v(s) \mathrm{d} s \in$ $\tilde{K}$, almost surely, we get for $t<T$,

$$
\begin{aligned}
V(t, s, y) \leq & \sup _{(\lambda, v) \in \Lambda \times \mathcal{D}} E^{Q^{\lambda, v}}\left[g\left(S^{0}(T) \exp \left(-\int_{t}^{T} v(s) \mathrm{d} s\right)\right)\right. \\
& \left.\times \exp \left(-\delta\left(\int_{t}^{T} v(s) \mathrm{d} s\right)\right) \mid\left(S^{0}, Y\right)(t)=(s, y)\right] \\
\leq & \sup _{(\lambda, v) \in \Lambda \times \mathcal{D}} E^{Q^{\lambda, v}}\left[\hat{g}\left(S^{0}(T)\right) \mid\left(S^{0}, Y\right)(t)=(s, y)\right] \\
= & \sup _{\lambda \in \Lambda} E^{Q^{\lambda, 0}}\left[\hat{g}\left(S^{0}(T)\right) \mid\left(S^{0}, Y\right)(t)=(s, y)\right] .
\end{aligned}
$$

We also want to prove the reverse inequality. Let $\left\{v_{k}\right\}$ be a maximizing sequence such that $g\left(s \mathrm{e}^{-v_{k}}\right) \mathrm{e}^{-\delta\left(v_{k}\right)} \rightarrow \hat{g}(s)$, and choose the constant controls $\mu_{k}(u)=v_{k} /(T-t)$ to get (for $t<T)$

$$
V(t, s, y) \geq \sup _{\lambda \in \Lambda} E^{Q^{\lambda, v_{k}}}\left[g\left(S^{0}(T) \mathrm{e}^{-v_{k}}\right) \mathrm{e}^{-\delta\left(v_{k}\right)} \mid\left(S^{0}, Y\right)(t)=(s, y)\right] .
$$

By standard estimates on diffusion processes, one concludes that $S^{0}(t)$ converges in $L^{2}$ to $S^{0}(T)$, as $t \rightarrow T$, therefore also almost surely along a subsequence. From this, by Fatou's lemma and lower semicontinuity of $g$, we get

$$
\liminf _{t \rightarrow T} V(t, s, y) \geq g\left(s \mathrm{e}^{-v_{k}}\right) \mathrm{e}^{-\delta\left(v_{k}\right)} .
$$

Taking the limit as $k \rightarrow \infty$ we get

$$
V(T-, s, y) \geq \hat{g}(s) .
$$

Therefore, in order to super-replicate $g(S(T)$ ), we have to super-replicate at least $\hat{g}(S(T)$ ), which implies that we can replace $g$ with $\hat{g}$ in (4.1). But then, repeating the arguments of (6.6) with $g$ replaced by $\hat{g}$, and using the fact that $H(\hat{g})=\hat{g}$ we see that, for $t<T$,

$$
\begin{aligned}
V(t, s, y) & =\sup _{(\lambda, v) \in \Lambda \times \mathbb{D}} E^{Q^{\lambda, v}}\left[\hat{g}(S(T)) \exp \left(-\int_{t}^{T} \delta(v(s)) \mathrm{d} s\right) \mid(S, Y)(t)=(s, y)\right] \\
& =\sup _{\lambda \in \Lambda} E^{Q^{\lambda, 0}}\left[\hat{g}\left(S^{0}(T)\right) \mid\left(S^{0}, Y\right)(t)=(s, y)\right] .
\end{aligned}
$$


In other words the supremum over the processes $v \in \mathscr{D}$ is obtained at $v(\cdot) \equiv 0$. But now we are back to the case without constraints, with payoff $\hat{g}$, and it follows that $V$ satisfies the Barenblatt PDE (6.1) with the terminal condition (6.4). In particular, $V=\tilde{V}$ is a classical solution to the HJB equation (4.16), hence $\left(v s V_{s}+\delta(v) V\right)(t, s) \geq 0$, for all $v \in \tilde{K},(t, s) \in$ $[0, T) \times \mathbb{R}_{+}^{*}$. By Theorem 13.1 in Rockafellar (1970), this implies that the portfolio (6.5) satisfies the constraints. Consequently, $V(0, S(0)) \geq U(0)$, hence $V(0, S(0))=U(0)$.

\section{Mixed case}

In this section we get similar results for the mixed case, in which Assumption 5.1 on the volatility holds, but its infimum is not necessarily zero.

\section{Assumption 7.1.}

$$
\inf _{y \in \mathbb{R}} \sigma(t, s, y)=\underline{\sigma}(t, s) \quad \text { for all }(t, s) \in[0, T) \times \mathbb{R}_{+}^{*},
$$

and $\underline{\sigma}$ satisfies the same assumptions as $\sigma$.

The following result holds for lower semicontinuous, non-negative and non-zero payoff functions $g$, under the assumptions of Sections 2 and 3.

Theorem 7.1. Under Assumptions 5.1, 6.2 and 7.1, and in the presence of constraints described by set $K=[l, u]$, the minimal super-replicating cost for the contingent claim $g(S(T))$ is given by $U(0)=\tilde{V}(0, S(0))$ where $\tilde{V}$ is the classical solution (if one exists) to the PDE

$$
\begin{aligned}
v_{t}+\frac{1}{2} s^{2} \underline{\sigma}^{2} v_{s s} & =0, \\
v(T, s) & =\tilde{g}(s)
\end{aligned}
$$

The corresponding super-replicating portfolio is given by

$$
\pi(t)=\frac{S(t) \tilde{V}_{s}(t, S(t))}{\tilde{V}(t, S(t)} .
$$

If there are no constraints $(K=\mathbb{R})$, then the same is valid even if Assumption 6.2 is not satisfied.

Proof. We only sketch the proof, since it is similar to those above. Again, we first assume that there are no constraints. By Lemma 5.1 we know that the function $V$ is concave. It also has to satisfy $V(T-, \cdot) \geq g(\cdot)$, therefore $V(T-, \cdot) \geq \tilde{g}(\cdot)$. Moreover, from (4.14) we see that $V$ is a viscosity supersolution to

$$
-v_{t}+\frac{1}{2} s^{2} \underline{\sigma}^{2} v_{s s}=0
$$

By the maximum principle we then get $V \geq \tilde{V}$, hence $U(0) \geq \tilde{V}(0, S(0))$. The reverse inequality is easy to show using Itô's rule on $\bar{V}$. The case with constraints is then proved as in the previous section. 


\section{Examples}

\subsection{European put options}

European put options are defined by the terminal payoff function

$$
g(s)=(\kappa-s)^{+}, \quad s \in \mathbb{R}_{+}^{*},
$$

for some $\kappa \geq 0$. Using the characterization of $\tilde{g}$ given in Lemma 5.5, and recalling that $K$ contains 0 , we see that $\tilde{g}(s)=\kappa$ and therefore $\tilde{g}^{\prime}(s)=0$. It follows that restriction (5.8) is satisfied. By Theorem 5.2 we have that, under Assumption 5.1, the super-replication cost of the European put option is given by

$$
U(0)=\kappa,
$$

and the corresponding super-replicating portfolio is $\tilde{\pi}(t)=0$ for all $t \in[0, T]$.

\subsection{European call options}

European call options are characterized by the terminal payoff function

$$
g(s)=(s-\kappa)^{+}, \quad s \in \mathbb{R}_{+}^{*} .
$$

We could get the super-replication cost directly, but we choose to use the put-call parity $s-\kappa=$ $(s-\kappa)^{+}-(\kappa-s)^{+}$, to transform the problem to the case of European put options. Assume first that there are no constraints, i.e. $l=-\infty$ and $u=+\infty(>1)$. Then from Proposition 3.1,

$$
\begin{aligned}
U(0) & \geq \sup _{\lambda \in \Lambda} E^{Q^{\lambda, 0}}\left[(S(T)-\kappa)^{+}\right] \\
& =\sup _{\lambda \in \Lambda} E^{Q^{\lambda, 0}}\left[(\kappa-S(T))^{+}+(S(T)-\kappa)\right] \\
& =S(0)-\kappa+\sup _{\lambda \in \Lambda} E^{Q^{\lambda, 0}}\left[(\kappa-S(T))^{+}\right],
\end{aligned}
$$

since the process $\{S(t), 0 \leq t \leq T\}$ is a martingale under $Q^{\lambda, 0}$ for any $\lambda \in \Lambda$. Therefore, applying the results of the previous section concerning European put options, we see that under Assumption 5.1, the super-replication cost of the European call option in the absence of portfolio constraints satisfies $U(0) \geq S(0)$. The reverse inequality is trivial (see also (5.7)). Therefore

$$
U(0)=S(0)
$$

Now, in the presence of constraints, this is obviously also the cost if $u \geq 1$, since one can still buy one share of the stock. If $u<1, \hat{g} \equiv \tilde{g} \equiv \infty$, and it is impossible to super-replicate the call (see Remark 5.4).

Remark 8.1. The above examples are extreme, in the sense that the super-replicating strategies are expensive and not very interesting from a practical point of view. The example below is less extreme because the volatility process is bounded. For more examples as well as numerical calculations see Broadie et al. (1998) and Avellaneda et al. (1995). 
TABLE 1: Numerical results for a range of initial stock prices and times to expiration.

\begin{tabular}{|c|c|c|c|c|c|c|}
\hline \multirow{3}{*}{$\begin{array}{c}\begin{array}{c}\text { Time to } \\
\text { expiration }\end{array} \\
\text { At expiration }\end{array}$} & \multicolumn{6}{|c|}{$\begin{array}{l}\text { Current Stock Price } \\
\text { Option price/hedge option }\end{array}$} \\
\hline & \multicolumn{2}{|c|}{1} & \multicolumn{2}{|c|}{1.5} & \multicolumn{2}{|c|}{2} \\
\hline & 0.25 & 2 & 0.5625 & 2 & 1 & 0.045295 \\
\hline 36 days to exp. & 0.251002 & 2 & 0.564754 & 1.999912 & 0.96833 & 0.071558 \\
\hline 72 days to exp. & 0.252008 & 2 & 0.566999 & 1.998106 & 0.94631 & 0.081278 \\
\hline 108 days to exp. & 0.253017 & 2 & 0.569167 & 1.992396 & 0.92982 & 0.088424 \\
\hline 145 days to exp. & 0.254031 & 1.999998 & 0.57119 & 1.982839 & 0.91669 & 0.093909 \\
\hline \multirow[t]{2}{*}{ Today } & 0.255049 & 1.999986 & 0.573029 & 1.970085 & 0.90572 & 0.099039 \\
\hline & \multicolumn{2}{|c|}{2.5} & \multicolumn{2}{|l|}{3} & & \\
\hline At expiration & 0.64 & -2.0064 & 0.44444 & -2.00445 & & \\
\hline 36 days to exp. & 0.64774 & -2.0053 & 0.44982 & -2.0045 & & \\
\hline 72 days to exp. & 0.6553 & -1.9896 & 0.45525 & -2.00452 & & \\
\hline 108 days to exp. & 0.66216 & -1.954 & 0.46075 & -2.00409 & & \\
\hline 145 days to exp. & 0.66812 & -1.9063 & 0.46629 & -2.0019 & & \\
\hline \multirow[t]{2}{*}{ Today } & 0.67231 & -1.8543 & 0.47181 & -1.99652 & & \\
\hline & \multicolumn{2}{|c|}{3.5} & \multicolumn{2}{|l|}{4} & & \\
\hline At expiration & 0.32653 & -2.0033 & 0.25 & -2.0025 & & \\
\hline 36 days to exp. & 0.33048 & -2.0033 & 0.253019 & -2.0025 & & \\
\hline 72 days to exp. & 0.33447 & -2.0033 & 0.256075 & -2.0026 & & \\
\hline 108 days to exp. & 0.33851 & -2.0034 & 0.259168 & -2.0026 & & \\
\hline 145 days to exp. & 0.3426 & -2.0034 & 0.262299 & -2.0026 & & \\
\hline Today & 0.34674 & -2.0032 & 0.265467 & -2.0026 & & \\
\hline
\end{tabular}

\subsection{A butterfly spread option}

We consider here an example in which the volatility is bounded between $\underline{\sigma}^{2}(t, s) \equiv 0.1$ and $\bar{\sigma}^{2}(t, s) \equiv 0.2$. To make things more interesting, we look at a payoff that is neither convex nor concave, given by

$$
g(s)=(s-1)^{+} \quad \text { if } s \leq 2 ; \quad g(s)=(3-s)^{+} \quad \text { if } s \geq 2 .
$$

Suppose that the constraints are given by the interval $K=[-2,2]$. We will calculate the price using Theorem 6.1. It is easy to see that

$$
\hat{g}(s)=s^{2} / 4 \quad \text { if } s \leq 2 ; \quad \hat{g}(s)=4 / s^{2} \quad \text { if } s \geq 2 .
$$

Numerical results are given in Table 1, for a range of initial stock prices and times to expiration. The values of the portfolio are called the 'hedge ratio'. There is a rounding error, that sometimes gives the portfolio values slightly outside of the set $K$. For comparison, Table 2 gives the Black-Scholes price of $g(S(T))$ without constraints, and with volatility assumed to be constant and equal to $\sigma^{2}=0.15$.

\section{Appendix A. The proof of Proposition 4.1}

We now show that $V$ is a supersolution, in the viscosity sense, of the Bellman equation in Proposition 4.1. This proof is standard and is essentially based on the dynamic programming 
TABLE 2: Black-Scholes price for a range of initial stock prices and times to expiration.

\begin{tabular}{|c|c|c|c|c|c|c|c|c|}
\hline \multirow{3}{*}{$\begin{array}{c}\begin{array}{c}\text { Time to } \\
\text { expiration }\end{array} \\
\text { At expiration }\end{array}$} & \multicolumn{8}{|c|}{$\begin{array}{c}\text { Current Stock Price } \\
\text { Option price/hedge option }\end{array}$} \\
\hline & \multicolumn{2}{|c|}{1} & \multicolumn{4}{|c|}{1.5} & \multicolumn{2}{|c|}{2} \\
\hline & 0 & 0 & 0.5 & & 3 & & 1 & 0 \\
\hline 36 days to exp. & 0.019943 & 25.41271 & 0.5014 & & 2.9913 & & 0.96156 & -0.02834 \\
\hline 72 days to exp. & 0.028204 & 18.05664 & 0.5020 & & 2.9878 & & 0.94563 & -0.03922 \\
\hline 108 days to exp. & 0.034543 & 14.82784 & 0.5024 & & 2.9850 & & 0.93341 & -0.05226 \\
\hline 145 days to exp. & 0.039887 & 12.87293 & 0.5028 & & 2.9827 & & 0.92311 & -0.05831 \\
\hline \multirow[t]{2}{*}{ Today } & 0.044595 & 11.53521 & 0.5032 & & 2.9807 & & 0.91404 & -0.06305 \\
\hline & \multicolumn{2}{|c|}{2.5} & \multicolumn{3}{|c|}{3} & \multicolumn{2}{|c|}{3.5} & \\
\hline At expiration & 0.5 & -5 & 0 & 0 & & 0 & 0 & \\
\hline 36 days to exp. & 0.49856 & -5.0145 & 0.0185 & & .9749 & 0 & 0 & \\
\hline 72 days to exp. & 0.49796 & -5.0205 & 0.02616 & & .269 & 0 & 0 & \\
\hline 108 days to exp. & 0.4975 & -5.0251 & 0.03204 & & .6697 & 0 & 0 & \\
\hline 145 days to exp. & 0.49711 & -5.029 & 0.037 & & .4488 & 0 & 0 & \\
\hline Today & 0.49677 & -5.0325 & 0.04137 & & .2151 & 0 & 0 & \\
\hline
\end{tabular}

principle. We refer to Crandall et al. (1992) or Fleming and Soner (1993) for definitions and standard estimates of the theory of viscosity solutions.

Lemma A.1 (Dynamic Programming Principle.) We have, for all $0<t<u<T$,

$$
V(t, s, y) \geq \sup _{(\lambda, \nu) \in \Lambda \times D} E^{Q^{\lambda, v}}\left[V\left(u, S_{t, s, y}(u), Y_{t, s, y}(u)\right) \exp \left(-\int_{t}^{u} \delta(v(r)) \mathrm{d} r\right)\right],
$$

where $\left\{\left(S_{t, s, y}(u), Y_{t, s, y}(u)\right), t \leq u \leq T\right\}$ is the process $(S, Y)$ satisfying (2.1-2.2) with the initial condition $(S(t), Y(t))=(s, y)$.

Proof. See Cvitanić and Karatzas (1993), Proposition 6.2 (notice that, although the authors assume bounded coefficients for the diffusion driving $(S, Y)$, their proof of the dynamic programming principle does not require such assumptions).

We first show that $V$ is lower semicontinuous. Let $\left(t^{n}, x^{n}, y^{n}\right)$ be a sequence such that $\left(t^{n}, s^{n}, y^{n}\right) \rightarrow(t, s, y)$. By standard estimates of diffusion processes (using the

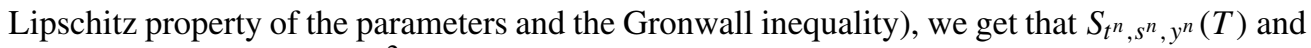
$Y_{t^{n}, s^{n}, y^{n}}(T)$ converge in $L^{2}$ to $S_{t, s, y}(T)$ and $Y_{t, s, y}(T)$, respectively. So, there exists a (relabeled) subsequence $\left(t^{n}, s^{n}, y^{n}\right)$ so that the above convergences are almost surely convergences. We have then, for a fixed $(\lambda, v) \in \Lambda \times \mathscr{D}$, by Fatou's lemma, and since $g$ is lower semicontinuous,

$$
\begin{aligned}
\liminf _{n} V\left(t^{n}, s^{n}, y^{n}\right) & \geq \liminf _{n} E^{\lambda, v}\left[g\left(S_{t^{n}, s^{n}, y^{n}}(T)\right) \exp \left(-\int_{t^{n}}^{T} \delta(v(r)) \mathrm{d} r\right)\right] \\
& \geq E^{\lambda, v}\left[\liminf _{n} g\left(S_{t^{n}, s^{n}, y^{n}}(T)\right) \exp \left(-\int_{t^{n}}^{T} \delta(v(r)) \mathrm{d} r\right)\right] \\
& \geq E^{\lambda, v}\left[g\left(S_{t, s, y}(T)\right) \exp \left(-\int_{t}^{T} \delta(v(r)) \mathrm{d} r\right)\right],
\end{aligned}
$$

and after taking the supremum over $(\lambda, \nu)$, we have completed this step of the proof. 
Next, fix $(t, s, y) \in[0, T) \times \mathbb{R}_{+}^{*} \times \mathbb{R}$ and consider a test function $\varphi \in C^{2}\left([0, T] \times \mathbb{R}_{+}^{*} \times \mathbb{R}\right)$ such that

$$
0=(V-\varphi)(t, s, y)=\min _{[0, T] \times \mathbb{R}_{+}^{*} \times \mathbb{R}}(V-\varphi) .
$$

By substituting $\varphi$ for $V$ (and $t+h$ for $u$ ) in the inequality of the previous lemma, we get

$$
\begin{gathered}
0 \geq \sup _{(\lambda, v) \in \Lambda \times \mathcal{D}} E^{Q^{\lambda, v}}\left[\varphi\left(t+h, S_{t, s, y}(t+h), Y_{t, s, y}(t+h)\right) \exp \left(-\int_{t}^{t+h} \delta(v(u)) \mathrm{d} u\right)\right. \\
-\varphi(t, s, y)],
\end{gathered}
$$

and by Itô's lemma, and standard estimates on the state process $(S, Y)$ under the controlled probability measure $Q^{\lambda, \nu}$, we see that

$$
\liminf _{h \rightarrow 0}\left\{\frac{1}{h} E^{Q^{\lambda, \nu}}\left[\int_{t}^{t+h} g^{\lambda, v} \varphi\left(u, S_{t, s, y}(u), Y_{t, s, y}(u)\right) \mathrm{d} u\right]\right\} \geq 0, \quad \text { for all }(\lambda, \nu) \in \Lambda \times \mathscr{D},
$$

where $g^{\lambda, v}$ is the differential operator appearing inside the infimum of (4.2). Therefore, we obtain

$$
\inf _{(\lambda, v) \in \mathbb{R} \times \tilde{K}} g^{\lambda, v} \varphi(t, s, y) \geq 0 .
$$

Finally, the boundary condition is obtained as follows. Considering $v=0$ as control in (4.1) and taking limits as $t$ approaches $T$, we see that

$$
\liminf _{t \nearrow T} V(t, s, y) \geq g(s), \quad s \in \mathbb{R}_{+}^{*},
$$

by Fatou's Lemma.

\section{Appendix B}

In this appendix, we provide conditions on the coefficients $\underline{\sigma}, \bar{\sigma}$ and the payoff function $g$ which guarantee the existence of a unique solution $\tilde{V}$ to the Barenblatt PDE (6.1). Consider the auxiliary optimal control problem

$$
\tilde{V}(t, s)=\sup _{\alpha \in \mathcal{C}} E\left[g\left(S^{\alpha}(T)\right) \mid S^{\alpha}(t)=s\right]
$$

where $\mathcal{C}$ is the set of all $\{\mathcal{F}(t)\}$-adapted processes valued in $[0,1]$ and $S^{\alpha}$ is the controlled process defined by the stochastic differential equation

$$
\frac{\mathrm{d} S^{\alpha}(t)}{S^{\alpha}(t)}=\tilde{\sigma}\left(t, S^{\alpha}(t), \alpha(t)\right) \mathrm{d} W(t)
$$

with

$$
\tilde{\sigma}(t, s, a)=(1-a) \underline{\sigma}(t, s)+a \bar{\sigma}(t, s) .
$$


The Bellman equation associated with this optimal control problem is given by

$$
v_{t}(t, s)+\frac{1}{2} \sup _{0 \leq a \leq 1}\left\{s^{2} \tilde{\sigma}^{2}(t, s, a) v_{s s}(t, s)\right\}=0,
$$

which is exactly the Barenblatt PDE (6.1). Furthermore, at the terminal date $T$, we have $\tilde{V}(T, s)=g(s)$.

Since the control set is bounded we can apply standard regularity results for the HJB equation (6.1). We denote by $C_{b}^{k}(\mathbb{R})$ the space of real-valued functions $\psi$ defined on $\mathbb{R}$ such that $\psi$ and its derivatives of order less than or equal to $k$ are continuous and bounded. The space $C_{b}^{k, m}([0, T] \times \mathbb{R})$ is defined similarly. The following assumptions are made.

(A1) There exists some $\varepsilon>0$ such that $\underline{\sigma}(t, s) \geq \varepsilon$ for all $(t, s) \in[0, T] \times \mathbb{R}_{+}^{*}$.

(A2) Let $\hat{\sigma}(t, x, a)=\tilde{\sigma}\left(t, \mathrm{e}^{x}, a\right)$. Then, for fixed $a \in[0,1], \hat{\sigma} \in C^{1,2}([0, T], \mathbb{R})$ and the functions $\hat{\sigma}, \hat{\sigma}_{t}, \hat{\sigma}_{x}$ and $\hat{\sigma}_{x x}$ are bounded in $[0, T] \times \mathbb{R} \times[0,1]$.

(A3) The function $\hat{g}: x \longmapsto g\left(e^{x}\right)$ lies in $C_{b}^{3}(\mathbb{R})$.

Then, under Assumptions (A1), (A2) and (A3), the value function $\tilde{V}$ is the unique solution of (6.1)-(6.2) in the class of functions $v$ such that $(t, x) \longmapsto v\left(t, \mathrm{e}^{x}\right)$ lies in $C_{b}^{1,2}([0, T], \mathbb{R})$ (see Theorems 4.2 and 4.4 in Fleming and Soner (1993)).

\section{Acknowledgements}

The research of the first author was partially supported by NSF grant \#DMS-95-03582. He is also grateful to CREST for its hospitality in July 1996, when this research was initiated.

We are grateful to Victoria Pikovsky for the numerical calculations in Section 8.3, to $\mathrm{H}$. Mete Soner for providing us with some 'viscosity tips', and to Yuri Kabanov for helpful comments that prompted the introduction of Lemma 4.1.

\section{References}

Avellaneda, M., Levy, A. And Paras, A. (1995). Pricing and hedging derivative securities in markets with uncertain volatilities. Appl. Math. Finance 2, 73-88.

Bergman, Y. Z., Grundy, B. D. And Wiener, Z. (1996). General properties of option prices. J. Finance 51, 1573-1610.

Broadie, M., Cvitanić, J. And Soner, M. (1998). Optimal replication of contingent claims under portfolio constraints. Rev. Financial Studies. 11, 59-79.

Buckdahn, R. AND Hu, Y. (1998). Pricing of American contingent claims with jump stock price and constrained portfolios. Math. Operat. Res. 23, 177-203.

Crandall, M. G., Ishit, H. and Lions, P. L. (1992). User's guide to viscosity solutions of second order partial differential equations. Bull. Amer. Math. Soc. 27, 1-67.

Cvitanić, J. and Karatzas, I. (1993). Hedging contingent claims with constrained portfolios. Ann. Appl. Prob. 3, 652-681.

El Karoui, N., Jeanblanc-Picqué, M. and Shreve, S. E. (1998). Robustness of the Black-Scholes formula. Math. Fianance 8, 93-126.

El Karoui, N. and Quenez, M. C. (1995). Dynamic programming and pricing of contingent claims in an incomplete market. SIAM J. Control Optim. 33, 29-66.

Fleming, W. H. And Soner, H. M. (1993). Controlled Markov Processes and Viscosity Solutions. Springer, New York.

Frey, R. AND Sin, C. A. (1999). Bounds on European option prices under stochastic volatility. Math. Fianance 9, 97-116.

Hull, J. And White, A. (1987). The pricing of options on assets with stochastic volatilities. J. Finance 42, 281300. 
Jouini, E. And Kallal, H. (1995). Arbitrage in securities markets with short-sales constraints. Math. Finance 3, 197-232.

Karatzas, I. and Kou, S. (1996). On the pricing of contingent claims under constraints. Ann. Appl. Prob. 6, 321-369.

Karatzas, I. And Shreve, S. E. (1991). Brownian Motion and Stochastic Calculus. Springer, New York.

Krylov, N. V. (1980). Controlled Diffusion Processes. Springer, Berlin.

Lions, P. -L. (1983). Optimal control of diffusion processes and Hamilton-Jacobi-Bellman equations, parts I and II. Commun. P.D.E. 8, 1101-1174 and 1229-1276.

Rockafellar, R. T. (1970). Convex Analysis. Princeton University Press, Princeton, NJ.

Wiggins, J. B. (1987). Option values under stochastic volatility: Theory and empirical estimates. J. Financial Economics 19, 351-372. 